\title{
Diacronie
}

Studi di Storia Contemporanea

$N^{\circ} 28,4 \mid 2016$

La voce del silenzio

\section{Neill Lochery, Brasil: os frutos da guerra}

\section{Dilton Cândido Santos Maynard}

Traduttore: Jacopo Bassi

\section{(2) OpenEdition}

\section{Journals}

\section{Edizione digitale}

URL: http://journals.openedition.org/diacronie/4675

DOI: 10.4000/diacronie.4675

ISSN: 2038-0925

\section{Editore}

Association culturelle Diacronie

\section{Notizia bibliografica digitale}

Dilton Cândido Santos Maynard, « Neill Lochery, Brasil: os frutos da guerra », Diacronie [Online], N²8, 4 ।

2016, documento 34, Messo online il 29 décembre 2016, consultato il 24 septembre 2020. URL :

http://journals.openedition.org/diacronie/4675; DOI : https://doi.org/10.4000/diacronie.4675 
N. 28 | 4|2016 La voce del silenzio: intelligence, spionaggio e conflitto nel XX secolo

34/

\title{
RECENSIONE:
}

\section{Neill LOCHERY, Brasil: os frutos da guerra, Rio de Janeiro, Intrínseca, 2015, 368 pp.}

\author{
a cura di Dilton Cândido Santos MAYNARD * \\ traduzione a cura di Jacopo BASSI
}

La Seconda guerra mondiale ha fornito al Brasile l'occasione per trasformarsi nella nazione più influente del Sudamerica e guadagnare spazio come protagonista nello scacchiere globale. Per questa ragione stringere alleanze con la Germania e gli Stati Uniti - le principali potenze nel periodo precedente il conflitto - era qualcosa di più che necessario: era strategico. Alla conclusione della guerra, tuttavia, sebbene si fosse optato per il blocco dei vincitori, i risultati non furono esattamente quelli sperati.

Stando così le cose, quali furono i risultati del coinvolgimento brasiliano nella Seconda guerra mondiale? Che cosa ottenne il paese a seguito dell'impegno profuso in quel periodo? Quale fu il ruolo di Getúlio Vargas (1882-1954) e del suo governo nelle trasformazioni delle infrastrutture, dell'economia, del settore militare e dell'immagine proposta al mondo dal Brasile? È questa la storia raccontata da Neill Lochery in Brasil: os frutos da Guerra.

Lochery è professore presso la University College London. È autore di alcuni bestsellers e collaboratore di diversi giornali e riviste, come «The Wall Street Journal» e «The Washington Post». Il suo libro è diviso in sei sezioni, per un totale di diciannove capitoli a cui fa seguito un epilogo. Una bella raccolta fotografica, composta da sedici scatti in bianco e nero arricchisce l'opera, che si compone di 367 pagine. Il repertorio di fonti utilizzate dall'autore è ampio e diversificato: diari, giornali, atti di processi, riviste, un'abbondante corrispondenza diplomatica (memorandum, telegrammi, etc.), discorsi e testimonianze raccolte negli archivi brasiliani, nordamericani e britannici.

L'opera di Lochery prende in esame il progetto sorto con la cosiddetta "politica di buon vicinato", concetto elaborato dal Segretario di Stato nordamericano Cordell Hull (1871-1955) e dal diplomatico Sumner Welles (1892-1961) e applicato da Franklin D. 
Roosevelt (1882-1945) per garantire l'adesione dei paesi latinoamericani allo sforzo bellico statunitense. È a partire da queste premesse che l'autore mette in relazione le pretese nordamericane di porsi come potenza egemonica del continente e le ambizioni di Getúlio Vargas e Oswaldo Aranha (1894-1960) di trasformare il Brasile ampliando la sua capacità industriale, rinforzando la sua economia e modernizzando le sue forze armate.

L’idea di fondo presentata nel libro è quella per cui il Brasile moderno sarebbe sorto negli anni della Seconda guerra mondiale. Questa prospettiva appare anche nel titolo originale dell'opera Brazil: The fortunes of War. World War II and the making of modern Brazil'. È quella secondo cui il conflitto globale avrebbe portato a mutamenti fondamentali nelle infrastrutture, nelle comunicazioni, nella cultura e nelle forze armate del paese, che si trasformò da «un angolo di mondo tropicale in un turbolento partecipante attivo, relativamente addentro alla geopolitica globale ${ }^{2}$.

La preoccupazione che il Brasile potesse allearsi con l'Asse, le conseguenze di una possibile ascesa dell'Argentina nello scenario regionale, i pericoli della presenza delle truppe nemiche in territorio americano erano alcune delle ragioni per cui gli Stati Uniti prestarono attenzione agli orientamenti della politica intrapresa da Vargas. Oltre a ciò, le colonie di immigranti tedeschi, italiani e giapponesi in terra brasiliana facevano sì che gli Stati Uniti e la Gran Bretagna fossero attente a come l'Estado Novo si muoveva nei confronti della Germania e, soprattutto, dell'Italia.

La simpatia dei militari d'alto grado per i successi militari tedeschi e gli elogi dei giuristi e degli altri intellettuali legati a Vargas nei confronti dello Stato fascista erano chiari indizi del fatto che il Brasile correva il rischio di posizionarsi con le forze dell'Asse. Oltre a questo, le forti relazioni commerciali stabilite con la Germania alimentavano ancora di più la possibilità di un allineamento di Getúlio con Hitler.

Durante questo periodo il Brasile riuscì ad entrare in possesso degli armamenti tedeschi, questione che scatenò un grave incidente diplomatico con la Gran Bretagna e rese evidente la necessità di un maggior investimento da parte degli Stati Uniti per contenere l'influenza tedesca. Se da un lato il testo mette in evidenza la forte pressione del corpo diplomatico nordamericano le cui pretese erano di assicurarsi l'egemonia sul Sudamerica, dall'altro viene mostrata anche la corrispettiva pressione esercitata da Getúlio Vargas per fornire all'Esercito equipaggiamenti moderni ed evidenzia come,

\footnotetext{
${ }^{1}$ LOCHERY, Neill, Brazil: The fortunes of War. World War II and the making of modern Brazil, London, Basic Books, 2014.

2 LOCHERY, Neill, Brasil: os frutos da guerra, Rio de Janeiro, Intrínseca, 2015, p. 265.
} 
seppure in momenti del tutto particolari, il Brasile riuscì a negoziare da pari a pari con lo "Zio Sam".

In qualche modo, Frutos da Guerra appare come una sorta di "storia intima" del Brasile, tanto grande è la leggerezza con cui Lochery presenta i momenti salienti che portarono alla creazione dell'Estado Novo - il tentativo di coup d'état e l'attacco degli integralisti al Palácio Guanabara, nel 1938, che viene trasformato quasi in un thriller -, la scelta del Brasile di sostenere gli Stati Uniti, le dispute tra le personalità principali che giravano intorno a Getúlio, la partecipazione effettiva del Brasile alla guerra con la creazione della Força Expedicionária Brasileira (FEB) e il suo invio sui campi di battaglia italiani nel 1944 e, infine, il graduale e malinconico epilogo del politico gaúcho. Tutto ciò viene affrontato in una prospettiva che contempla la tensione fra il privato e il pubblico. I diari, gli appunti, le lettere e le testimonianze confidenziali vengono esplorate con bramosia dall'autore.

Lochery conduce il suo lettore attraverso i corridoi del Palácio da Guanabara, sale con lui le scale ed entra in punta di piedi nello studio di Vargas, cammina con lui attraverso i campi da golf, osserva i disegni formati dagli sbuffi di sigaro del leader brasiliano. Non pago, accompagna il dittatore ed altre personalità in passeggiate riflessive, in viaggi in automobile con vista sulla spiaggia di Copacabana o nel piacevole clima di montagna che si incontra a Petrópolis, dove i cariocas si rifugiavano per sfuggire al caldo intenso tra gennaio e febbraio.

Getúlio Vargas, sua figlia Alzira (1914-1992) - definita da Waldo Frank (1889-1967) «l'occhio destro di Vargas» -, il Ministro degli Affari Esteri Oswaldo Aranha - definito, sempre da Frank, l'«occhio sinistro di Vargas» -, il Ministro della Guerra, il Generale Góes Monteiro (1889-1956), il Capo di Stato Maggiore dell'Esercito, il Generale Dutra (1883-1974), oltre ai rappresentanti degli Stati Uniti in Brasile come Sumner Welles, Jefferson Caffery (1886-1974), Orson Welles (1915-1985) e Nelson Rockfeller (19081979), sono i principali personaggi di una narrazione inizialmente marcata dalle tensioni del nuovo regime creato da Vargas, che passa dall'euforia per i possibili accordi e i ringraziamenti diplomatici al Brasile per aver autorizzato la realizzazione di basi nordamericane sul territorio nazionale fino alle due "cadute" di Vargas, nel 1945 e nel 1954. Per quest'ultima l'autore offre un racconto dettagliato e drammatico dei momenti che precedettero il suicidio dell'uomo conosciuto come il "Padre dei poveri".

Ciononostante, alcune rettifiche sono necessarie. Lochery definisce il genero di Vargas come "governatore" di Rio de Janeiro. Ora, una volta istituito l'Estado Novo, erano in carica "interventores federais". Benché la funzione, in pratica fosse sostanzialmente la stessa, la definizione e il modo di accedere alla carica erano 
differenti. Troviamo anche passaggi che necessiterebbero di una migliore spiegazione quando, ad esempio, l'autore scrive che i soldati della FEB «inventarono persino un motto per [definire] loro stessi: "il serpente che fuma"» 3, ma senza spiegarne le ragioni ${ }^{4}$. In un testo tanto attento ai dettagli, una dimenticanza come questa risulta strana, eccessiva.

Un altro aspetto che attira l'attenzione è il soffermarsi dell'autore nella narrazione dei siluramenti delle navi mercantili brasiliane avvenuti nell'agosto del 1942. Questi attacchi costituirono la ragione formale in base a cui Vargas dichiarò ufficialmente guerra all'Italia e alla Germania. Lochery tratta degli attacchi, ma senza la precisione che contraddistingue l'opera nella maggior parte dei suoi passaggi. Come spiegare questa asimmetria di rigore scientifico?

Talvolta una delle ragioni per le reticenze summenzionate trova una giustificazione nel fatto che l'autore ricalca una tradizione poco stimolante della storiografia: quella di concentrare l'attenzione sostanzialmente sulle opere realizzate nella regione del Sudeste del Brasile. La conseguenza è che l'opera, in alcuni passaggi, finisce per tralasciare informazioni utili sul resto del paese in quell'epoca. Natal, ad esempio città che giocò un ruolo strategico nel periodo - appare solo brevemente nel testo. Lo stesso si può dire di Salvador, di Recife e, allo stesso modo, di Porto Alegre ${ }^{5}$, sebbene quest'ultima venga menzionata con un certo risalto all'inizio dell'opera.

L'effetto di questa interpretazione modellata su testi che già sono stati oggetto di dibattito - non necessariamente superati - produce un'analisi del Brasile durante la Seconda guerra mondiale soprattutto a partire dagli eventi di Rio de Janeiro. Risulta

\footnotetext{
3 Ibidem, p. 253.

${ }_{4}^{4}$ L'espressione indica qualcosa difficilmente realizzabile; venne impiegata nel 1944 in relazione a quella che veniva ritenuta un'improbabile entrata in guerra del Brasile. I giornalisti ritenevano che "fosse più facile che un serpente iniziasse a fumare, piuttosto che il Brasile entrasse in guerra". Quando la FEB intervenne in Italia - partecipando attivamente alla guerra di liberazione sull'Appennino tosco-emiliano - adottò come simbolo un serpente che fuma la pipa e come motto "A cobra vai fumar». Sul tema si veda: BERNADOTTI, Maria Adriana, CASALI, Luciano, Brasiliani e partigiani. L’immagine della Resistenza nella memorialistica brasiliana sulla Seconda guerra mondiale, in ARBIZZANI, Luigi (a cura di), Al di qua e al di là della Linea Gotica. 1944-1945: aspetti sociali, politici e militari in Toscana e in Emilia-Romagna, Firenze, Regioni Emilia-Romagna e Toscana, 1993, pp. 533-551; MORIGI, Massimo, SALMI, Stefano, Quando il cobra fumò. Una riflessione sulla forza di spedizione brasiliana a fianco degli alleati in Italia, in DALLA CASA, Brunello, PRETI, Alberto (a cura di), La montagna e la guerra, Bologna, Aspasia, 1999, pp. 373-386. Sull'argomento si veda anche il film: FERRAZ, Vicente, Road 47, Primo Filmes - Stopline Films - Três Mundos Produções Verdeoro, Brasile-Portogallo, Italia, 107' [N.d.T.].

5 Si vedano ad esempio: PEDREIRA, Flávia Sá, Chiclete eu misturo com banana: Carnaval e cotidiano de guerra em Natal (1920-1945), Natal, Editora da UFRN, 2005; SCHURSTER, Karl, A Guerra como metáfora: aspectos da propaganda do Estado Novo em Pernambuco (19421945), Tesi discussa nella UFRPE, Recife, 2008; HAUSSEN, Doris Fagundes, Rádio e política: tempos de Vargas e Perón, Porto Alegre, EDIPUCRS, 2001.
} 
abbastanza strano anche il poco risalto concesso al ruolo del Departamento de Imprensa e Propaganda (DIP), soprattutto agli sforzi di Lourival Fontes (1899-1967) per creare una mitologia della figura di Vargas. Dall'opera, l'impressione che si ricava è che Vargas, contrariamente a ciò che afferma la storiografia più recente, divenne un mito politico unicamente ed esclusivamente grazie alle sue forze.

Il testo finisce così per adottare una lettura quasi idealizzata di Vargas, che viene deresponsabilizzato per la legislazione dell'Estado Novo. Minimizzata nell'opera, l'immensa repressione dell'epoca viene attribuita ai "simpatizzanti dell'Asse", come ad esempio il sinistro capo della polizia del Distretto Federale, Filinto Müller (1900-1973), ma non sostenuta dagli uomini a capo dell'Estado Novo. Basandosi su documenti come i diari dello stesso Getúlio Vargas o sulle memorie di Alzira - l'autore sembra sedotto dalla prospettiva che il presidente cercò di imporre sulla sua persona. Poco o nessuno spazio viene concesso per un approfondimento della personalità del politico gaúcho e di alcune polemiche sorte intorno al suo governo, come l'imprigionamento e la deportazione della militante comunista di origini ebraiche Olga Benário, incinta, verso la Germania nazista, che sono tralasciate dal testo.

Si nota che l'opera presenta passaggi talvolta ripetitivi, problema che merita di essere corretto in una prossima edizione. Questo avviene, ad esempio, tra le pagine 250 e 251, dove in un intervallo inferiore a quattro paragrafi per ben due volte si informa che le armi dei soldati della FEB «cadevano a pezzi nelle loro mani» ${ }^{6}$. Lo stesso avviene con l'indicazione del posizionamento delle truppe tedesche in Italia «su terreni elevati» 7 .

Al contempo, tuttavia, il libro presenta una certa difficoltà nell'analizzare alcuni caratteristiche del periodo, dal momento che non si sofferma su un aspetto, come fa ad esempio Antônio Pedro Tota guardando alle relazioni Brasile-Stati Uniti in campo culturale con il suo $O$ Imperialismo Sedutor ${ }^{8}$, o su un'analisi della propaganda in Brasile e in Argentina, sul modello di Maria Helena Capelato in Multidões em Cena ${ }^{9}$, sull'operato della FEB come Francisco Ferraz in Os Brasileiros e a Segunda Guerra Mundial $^{10}$ o ancora sull'ethos fascista del modello di Stato realizzato nel 1937 da Vargas, come Alcir Lenharo in Sacralização da Política ${ }^{11}$. In conseguenza di ciò si ha

${ }^{6}$ LOCHERY, Neill, Brasil: os frutos da guerra, cit., p. 251.

7 Ibidem, p. 278.

8 TOTA, Antonio Pedro, Imperialismo Sedutor: a americanização do Brasil, na época da Segunda Guerra, São Paulo, Companhia das Letras, 2000.

9 CAPELATO, Maria H. R. Multidões em cena: Propaganda política no varguismo e no peronismo, Campinas, Papirus, 1998.

${ }^{10}$ FERRAZ, Francisco César Alves, Os brasileiros e a Segunda Guerra Mundial, Rio de Janeiro, Jorge Zahar, 2005.

${ }^{11}$ LENHARO, Alcir, A sacralização da política, Campinas, Papirus, 1986. 
l'impressione di un'opera ben concepita, a prescindere dal fatto che sia ampia, molto ben scritta, ma in cui l'analisi perde spazio in favore di un racconto quasi privo di compromessi.

In definitiva Brasil: Frutos da Guerra funziona molto bene come opera di carattere generale. Nel coprire un periodo tanto ampio come quello intercorso fra i primi passi dell'Estado Novo e il suicidio di Vargas, Lochery dimostra un'invidiabile capacità di sintesi. Per via del suo testo piacevole da leggere, l'opera merita di essere letta non solamente dal pubblico degli addetti ai lavori. E per il suo tono intimista potrà certamente ispirare qualche film, telenovela, miniserie o qualcosa di simile. La passione dell'autore per il Brasile e per questo periodo permea ogni pagina; la sua ammirazione per Vargas, Alzira e Oswaldo Aranha è innegabile. Questa è una storia sincera dell'allora nascente Brasile moderno la maggior virtù e, per converso, il maggior problema dell'opera. 


\section{* L'autore}

Dilton Cândido Santos Maynard è professore presso il Dipartimento di Storia dell'Universidade Federal de Sergipe e insegna nel corso di Laurea magistrale in Scienze della Formazione e nel corso di laurea specialistica in Storia. È Professor Colaborador nel corso di laurea magistrale in Storia comparata dell'Universidade Federal do Rio de Janeiro (PPGHC/UFRJ). Coordina il Grupo de Estudos do Tempo Presente.

URL: < http://www.studistorici.com/progett/autori/\# Maynard >

\section{Per citare questo articolo:}

MAYNARD, Dilton Cândido Santos, «Recensione: Neill LOCHERY, Brasil: os frutos da guerra, Rio de Janeiro, Intrínseca, 2015, 368 pp.», Diacronie. Studi di Storia Contemporanea : La voce del silenzio: intelligence, spionaggio e conflitto nel XX secolo, 29/12/2016,

URL:< http://www.studistorici.com/2016/12/29/maynard_numero_28/ >

Diacronie Studi di Storia Contemporanea $\beta$ www.diacronie.it

Risorsa digitale indipendente a carattere storiografico. Uscita trimestrale.

redazione.diacronie@hotmail.it

Comitato di redazione: Jacopo Bassi - Luca Bufarale - Antonio César Moreno Cantano - Deborah Paci - Fausto Pietrancosta - Alessandro Salvador - Matteo Tomasoni - Luca Zuccolo

Diritti: gli articoli di Diacronie. Studi di Storia Contemporanea sono pubblicati sotto licenza Creative Commons 3.0. Possono essere riprodotti e modificati a patto di indicare eventuali modifiche dei contenuti, di riconoscere la paternità dell'opera e di condividerla allo stesso modo. La citazione di estratti è comunque sempre autorizzata, nei limiti previsti dalla legge. 